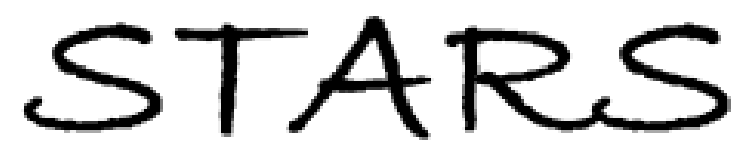

University of Central Florida

STARS

$1-1-2005$

\title{
Analytical expression for the output voltage of the triple resonance Tesla transformer
}

John Randolph Reed

University of Central Florida

Find similar works at: https://stars.library.ucf.edu/facultybib2000 University of Central Florida Libraries http://library.ucf.edu

This Article is brought to you for free and open access by the Faculty Bibliography at STARS. It has been accepted for inclusion in Faculty Bibliography 2000 s by an authorized administrator of STARS. For more information, please contactSTARS@ucf.edu.

\section{Recommended Citation}

Reed, John Randolph, "Analytical expression for the output voltage of the triple resonance Tesla transformer" (2005). Faculty Bibliography 2000s. 5579.

https://stars.library.ucf.edu/facultybib2000/5579

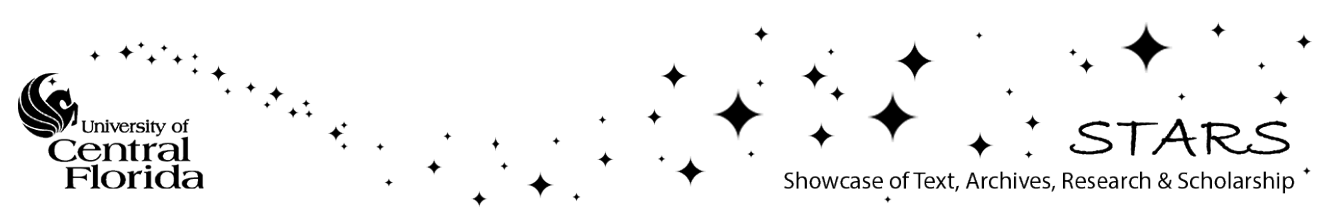




\title{
Analytical expression for the output voltage of the triple resonance Tesla transformer
}

\author{
John Randolph Reed \\ Department of Engineering and Computer Science, The University of Central Florida, \\ 400 Central Florida Boulevard, Orlando, Florida 32816
}

(Received 4 January 2005; accepted 5 September 2005; published online 11 October 2005)

\begin{abstract}
An analytical expression for the time-dependent output voltage of the triple resonance Tesla transformer is presented. Presently there does not exist any closed-form solution for the output voltage, and the investigators must use circuit simulators to examine the transformer's performance. Such simulators are satisfactory in many regards, but they cannot furnish physical insight into the triple resonance device. A governing equation provides this needed insight and opens the way for obtaining optimal high-performance transformers. The present analysis treats the transformer as three oscillatory $L C$ tank circuits feeding a load capacitance. The circuit is assumed to have a very high $Q$, which is a fundamental design practice for these pulsed power supplies. The formula is exercised using a known triple resonance circuit, and the results closely agree with the analysis of an industry standard circuit simulator. (C) 2005 American Institute of Physics.
\end{abstract}

[DOI: 10.1063/1.2093764]

\section{INTRODUCTION AND PREVIOUS WORK}

The Tesla transformer appears in two forms for highpower pulse mode operation. The first is the well-known "dual resonance" transformer where the two coexisting modal frequencies appear in the ratio of 1:2. This frequency ratio forces the alignment of the voltage maxima at a specific instant in time. Some of the useful mathematical and experimental discussions of this circuit are found in Refs. 1-4.

The second is the less-known form referred to as the "triple resonance" transformer. Tesla invented the triple resonance transformer, and a patent ${ }^{5}$ was issued for the same in 1913 (applied for in 1902).

Figure 1 shows the triple resonance transformer circuit under examination in this analysis. This pulse transformer circuit has three coexisting modal frequencies. The triple resonance device is currently of interest due to the search for high-performance pulse power production. Highperformance pulse transformers of this family could be arbitrarily defined as those obtaining voltage gains over 40 . The main literature of interest concerning the triple resonance device appears in the works of Tesla, ${ }^{5}$ Bieniosek, ${ }^{6-8}$ and de Queiroz. $^{9,10}$

The recent investigations into improvement in voltage gain have been in employing the triple resonance circuit possessing the modal frequency ratio of 1:2:3. This frequency ratio forces the alignment of the voltage maxima of the three oscillations, as in the dual resonance device, at a particular instant in time. This frequency ratio will be used in exercising the expression for output voltage. The governing equation for output voltage is not limited to any particular modal frequency ratio.

\section{OPERATION OF TRIPLE RESONANCE TRANSFORMER}

Expository ease is obtained by viewing the operation of the transformer in light of energy and capacitance relationships. This energy concept shows how the transformer reaches such extreme voltages during its pulse, and obtains higher voltage gains (per pound) than the dual resonance device. Examine Fig. 1 and note that after the primary capacitance discharges, the circuit undergoes a complicated transient, and the primary capacitor's energy is transferred across the magnetic link via mutual induction. The currents null, at this instant, and the voltage across the circuit peaks. The transformer, at this time, is storing the energy statically in the series string of self-capacitances. For the sake of clarity assume that each self-capacitance in the string is to be the same size, $C$. The triple resonance transformer has an extra inductor, with its associated self-capacitance; therefore its series string possesses three capacitances, including the load capacitance. The total series capacitance is lower than what would be obtained in a dual resonance device with its two series capacitances. The self-capacitances of the secondary inductor and the load capacitance would give the dual resonance string a total of $C / 2$ units of capacitance. However, the triple resonance string presents a total of only $C / 3$ units for energy storage $\left(1 / C_{\text {total }}=1 / C+1 / C+1 / C\right)$. This smaller total capacitance produces the desired higher peak voltages when storing the same amount of energy in the string. Several megavolts are typically obtained. The very large voltage in the system is why $C_{1}$ is the only convential capacitor. Its initial voltage is only about $15 \mathrm{kV}$. The self-capacitances of the inductors are able to support large voltages due to their large radii and turn-to-turn voltage grading.

The load capacitance is typically the electrostatic capacity of the corona shield of the electron gun. The peak voltage across the load capacitance occurs in the second half-cycle of 


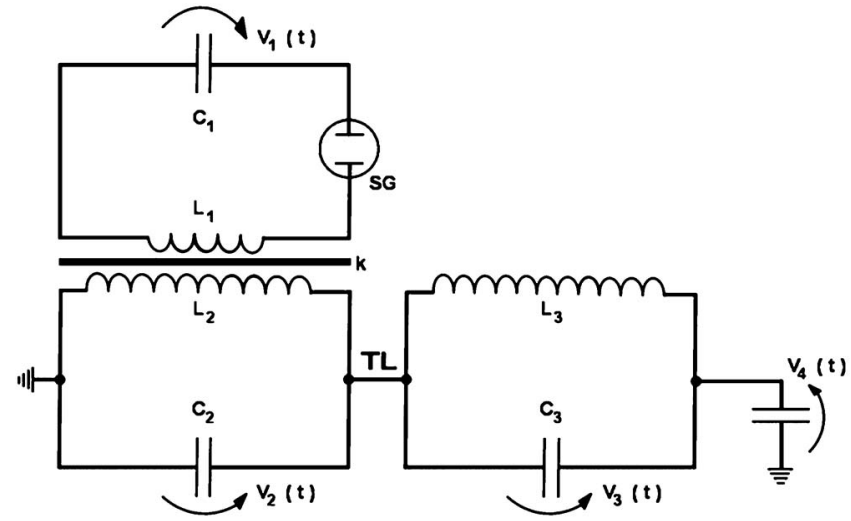

FIG. 1. Circuit of the high- $Q$ triple resonance Tesla transformer. $L_{1}$ and $C_{1}$ are the inductance and capacitance of the primary tank circuit. SG is a spark-gap switch. $L_{2}$ and $C_{2}$ are the inductance and self-capacitance of the secondary inductor. The coefficient of magnetic coupling between the primary circuit and the secondary circuit is $k . L_{3}$ and $C_{3}$ are the inductance and self-capacitance of the third inductor. The third inductor is treated as a parallel tank circuit with its base connected to the output side of the secondary inductor by transmission line TL. The third inductor feeds the load capacitance $C_{4}$. The oscillations within the circuit are initiated when the energy stored in $C_{1}$ is conducted into the circuit by commutation at the spark-gap switch. The time-dependent voltages across the primary circuit, secondary circuit, third inductor, and load capacitance during the oscillation are represented by $V_{i}(t), i=1-4$.

oscillation, for couplings between 0.6 and $0.7 .^{3}$ The capacitances are charged by three distinct oscillations wherein the current waves lag the voltage waves as the circuit is predominately an inductive one. The energy is taken, an instant later, from the load capacitance by any of the various forms of gating devices ${ }^{3}$ for the application at hand. "Load" is not an adequate descriptor as one is led to believe that the energy is being used to perform a final external task, when it is simply being stored, waiting to be taken. A better descriptor would be "terminal energy store." This description would fit with the often used "primary energy store," in reference to the energy stored in the primary capacitance before it is taken.

\section{PRESENTATION OF THE ANALYTICAL EXPRESSION FOR OUTPUT VOLTAGE}

The closed-form equation was developed by the method of the Laplace transform. ${ }^{11-13}$ The equation is later applied to a triple resonance pulse transformer circuit that possesses the frequency ratio of 1:2:3, and compared to the analysis of an industry standard circuit simulator ${ }^{14}$ and the voltage output of a real system. ${ }^{6}$

The symbols and their respective circuit components are found in Fig. 1, otherwise they are defined in the text.

The squares of the decoupled angular resonance frequencies of the tank circuits of Fig. 1 are defined as

$$
\omega_{1}^{2}=1, \quad \omega_{2}^{2}=\left(L_{1} C_{1}\right) /\left(L_{2} C_{2}\right),
$$

and

$$
\omega_{3}^{2}=\left(L_{1} C_{1}\right) /\left(L_{3} C_{3}\right) .
$$

The decoupled resonance frequencies are nondimensionalized by dividing by $\omega_{1}{ }^{2}$, and later the governing equation will be dimensioned based upon the units chosen for the
TABLE I. Data for the example triple resonance transformer. This transformer was designed using the frequency equation for the triple resonance transformer circuit. The modal frequencies in the coupled system, $f_{i}$, where $i=1-3$, are in the ratio of $1: 2: 3$.

\begin{tabular}{ccc}
\hline \hline Constant & Value & Unit \\
\hline$C_{1}$ & 0.299 & Microfarad \\
$C_{2}$ & 0.0001575 & Microfarad \\
$C_{3}$ & 0.0000255 & Microfarad \\
$C_{4}$ & 0.000060 & Microfarad \\
$L_{1}$ & 0.87 & Microhenry \\
$L_{2}$ & 639.9 & Microhenry \\
$L_{3}$ & 820.0 & Microhenry \\
$k_{1}$ & 0.666 & Hertz \\
$f_{1}$ & 270911.0 & Hertz \\
$f_{2}$ & 541823.0 & Hertz \\
$f_{3}$ & 812735.0 & \\
Gain & $\sim 50: 1$ & \\
\hline \hline
\end{tabular}

analysis. The units that were chosen to work with are microhenry and microfarad. These units were chosen for convenience in the computer program. The results of the analysis are dimensioned by multiplying by a proper form of $\omega_{1}^{2}$. The dimensioning of the governing equation will be addressed during the inverse of its transformation, at the end of this section.

The governing equation is the result of a Laplace transform analysis, which is given in Ref. 15 .

The presentation of the governing equation is facilitated by defining certain segments of its algebraic structure in the manner given next. The values of all the parameters are in Tables I and II.

Now define:

$$
\begin{aligned}
& e=\left(1+C_{4} / C_{2}+C_{4} / C_{3}\right), \\
& f=\left(1-k^{2}\right)\left(1+C_{4} / C_{2}+C_{4} / C_{3}\right), \\
& g=\left(1+C_{4} / C_{3}\right), \\
& h=\left(1+C_{4} / C_{2}\right), \\
& a_{1}=\left[e \omega_{1}^{2}+g \omega_{2}^{2}+\left(1-k^{2}\right) h \omega_{3}^{2}\right] / f,
\end{aligned}
$$

TABLE II. Constant data for the governing equation derived from the data of the example transformer. Note that $\Omega_{1}: \Omega_{2}: \Omega_{3}$ is in the ratio of 1:2:3.

\begin{tabular}{lccc}
\hline \hline Constants & Values & Constants & \multicolumn{1}{c}{ Values } \\
\hline$\omega_{1}{ }^{2}$ & 1.0 & $s_{1}{ }^{2}$ & -0.75415516 \\
$\omega_{2}{ }^{2}$ & 2.5794203 & $s_{2}{ }^{2}$ & -3.0162438 \\
$\omega_{3}{ }^{2}$ & 12.440459 & $s_{3}{ }^{2}$ & -6.7900988 \\
$e$ & 3.7336518 & $\Omega_{1}$ & 0.86842108 \\
$f$ & 2.077568 & $\Omega_{2}$ & 1.7367337 \\
$g$ & 3.3529412 & $\Omega_{3}$ & 2.6057818 \\
$h$ & 1.3807107 & $V_{0}$ & $1000.0 \mathrm{~V}$ \\
$a_{1}$ & 10.560498 & $W$ & 1960671.2 \\
$a_{2}$ & 27.876097 & $f_{1}$ & $270991.0 \mathrm{~Hz}$ \\
$a_{3}$ & 15.445545 & $f_{2}$ & $541949.0 \mathrm{~Hz}$ \\
$V_{P}$ & 225.26 & $f_{3}$ & $813135.0 \mathrm{~Hz}$ \\
$C_{\mathrm{I}}$ & -0.855897 & $C_{\mathrm{II}}$ & 1.103952 \\
$C_{\mathrm{III}}$ & -0.248053 & & \\
\hline \hline
\end{tabular}




$$
\begin{aligned}
& a_{2}=\left(g \omega_{1}^{2} \omega_{2}^{2}+h \omega_{1}^{2} \omega_{3}^{2}+\omega_{2}^{2} \omega_{3}^{2}\right) / f, \\
& a_{3}=\omega_{1}^{2} \omega_{2}^{2} \omega_{3}^{2} / f .
\end{aligned}
$$

Form the sixth-order polynomial in " $s$ " using the constants $a_{1}, a_{2}$, and $a_{3}$ as the coefficients from the above equations, resulting in

$$
s^{6}+a_{1} s^{4}+a_{2} s^{2}+a_{3}=0 .
$$

Equation (9) is now treated as a cubic in $s^{2}$. Let the unknown roots of Eq. (9) be defined as $-s_{1}{ }^{2},-s_{2}{ }^{2}$, and $-s_{3}{ }^{2}$ (see Ref. 13). It is now emphasized that the roots must be negative or the solution is not useful.

Define the following positive parameters as

$$
\Omega_{1}=\left(-s_{1}^{2}\right)^{1 / 2}, \quad \Omega_{2}=\left(-s_{2}^{2}\right)^{1 / 2}, \quad \text { and } \Omega_{3}=\left(-s_{3}^{2}\right)^{1 / 2} \text {. }
$$

The time-dependent output voltage $V_{4}(t)$ is the inverse Laplace transform of $P_{4}(s)$, as developed in Ref. 15 and given as

$$
\begin{aligned}
V_{4}(t)= & {\left[V_{0} k\left(L_{2} / L_{1}\right)^{1 / 2} \omega_{2}{ }^{2} / f\right]\left(\left\{\left(\Omega_{1}{ }^{2}-\omega_{3}{ }^{2}\right) /\left[\left(\Omega_{1}{ }^{2}-\Omega_{2}{ }^{2}\right)\right.\right.\right.} \\
& \left.\left.\times\left(\Omega_{1}{ }^{2}-\Omega_{3}{ }^{2}\right)\right]\right\} \cos \left(\Omega_{1} W t\right)+\left\{\left(\Omega_{2}{ }^{2}-\omega_{3}{ }^{2}\right) /\left[\left(\Omega_{2}{ }^{2}\right.\right.\right. \\
& \left.\left.\left.-\Omega_{1}{ }^{2}\right)\left(\Omega_{2}{ }^{2}-\Omega_{3}{ }^{2}\right)\right]\right\} \cos \left(\Omega_{2} W t\right)+\left\{\left(\Omega_{3}{ }^{2}\right.\right. \\
& \left.\left.\left.-\omega_{3}{ }^{2}\right) /\left[\left(\Omega_{3}{ }^{2}-\Omega_{1}{ }^{2}\right)\left(\Omega_{3}{ }^{2}-\Omega_{2}{ }^{2}\right)\right]\right\} \cos \left(\Omega_{3} W t\right)\right) .
\end{aligned}
$$

Equation (11) can be written in a compact form by defining the first factor as the voltage parameter $V_{P}$ and each constant coefficient of its respective cosine term as $C_{\mathrm{I}}, C_{\mathrm{II}}$, and $C_{\mathrm{III}}$. Furthermore, $W$, to dimension $V_{4}(t)$, is equal to $10^{6} /\left(L_{1} C_{1}\right)^{1 / 2}$ in order to obtain hertz because the units chosen for inductance and capacitance are microhenry and microfarad. $W$ also has a denominator of $\left(L_{1} C_{1}\right)^{1 / 2}$ as $\Omega_{i}^{2}$ inverts to $\Omega_{i}$ as the argument of the cosine functions, with $i=1-3$. The last statement (A-15) in Ref. 15 shows the particular inverse transformation to derive the denominator of $W$. The unit of time $t$ in Eq. (11) is in seconds. $V_{0}$ is the initial voltage of the primary energy storage capacitor $C_{1}$ in the units of volts. Note that $2 \pi f_{i}=\Omega_{i} W$, where $i=1-3$, from which the three modal frequencies can be computed. These frequencies will be in the ratio of 1:2:3 for the example transformer. Equation (11) can also be written as a voltage gain by removing the initial voltage $V_{0}$ from the voltage parameter, yielding:

$$
\begin{aligned}
V_{4}(t) / V_{0}= & V_{P}\left[C_{\mathrm{I}} \cos \left(2 \pi f_{1} t\right)+C_{\mathrm{II}} \cos \left(2 \pi f_{2} t\right)\right. \\
& \left.+C_{\mathrm{III}} \cos \left(2 \pi f_{3} t\right)\right] .
\end{aligned}
$$

The three cosine terms model the three coexisting modal oscillations within the circuit, and are maximum, \pm 1 , when their arguments are $-\pi$ and $2 \pi$ radians in time passing. The voltage peaks across $C_{4}$ at time: $t=\pi /\left(2 \pi f_{1}\right)$. This does not imply that their coefficients or the voltage parameter of the expression is also a maximum (or even large). These later relationships must be investigated to obtain an optimized circuit and maximize the output voltage.

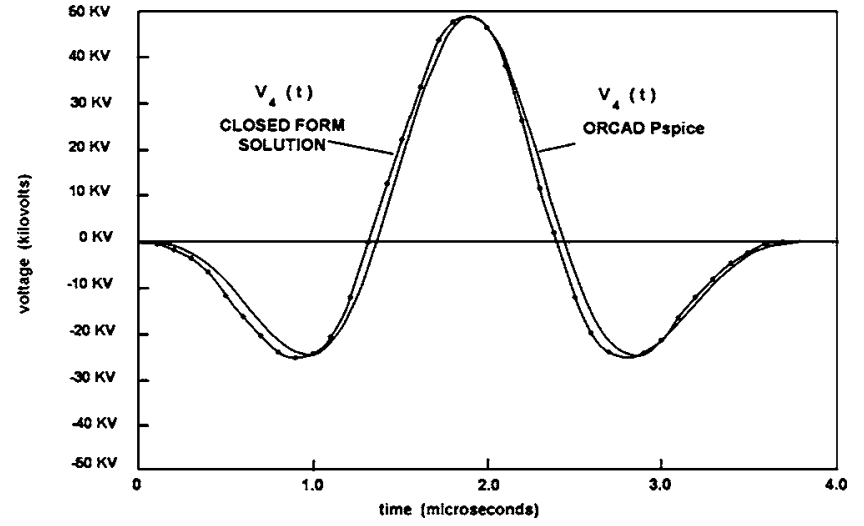

FIG. 2. Plot of the output voltage of the governing equation vs the output from the circuit simulator program. Each point is $0.1 \mu$ s time step in the governing equation.

\section{SOLUTION OF THE ANALYTICAL EXPRESSION}

This section will illustrate the use of the governing equation by installing the required data from a known triple resonance transformer. The transformer used in this example possesses a modal frequency ratio of 1:2:3. The example transformer also has a self-capacitance, $C_{3}$, for the third inductor, as all inductors have a resonance frequency. The required data are given in Tables I and II.

A True BASIC (Ref. 16) computer program exercised the governing equation for the example triple resonance Tesla transformer. The program is deposited in Ref. 15. The program is complete with an automatic root finder and print/plot statements that can be enabled or disabled at will. The time step in the program marched along at $1 / 10$ of a microsecond evaluating Eq. (11).

Figure 2 is the pulse wave form generated by the program exercising the governing equation.

\section{RESULTS}

Once all the constants from Tables I and II are substituted into Eq. (11) the only variable is time.

Figure 2 is a plot of the output of the closed-form solution compared with the output from the PSPICE (Ref. 14) analysis. The results compare very well. The results from the governing equation versus the results from the industry standard circuit simulator are practically congruent if they are time shifted by approximately $1 / 20$ th of a microsecond. This small time shift is due to a built in delay of switch closure in the circuit simulator program.

\section{CONCLUSIONS}

The closed-form solution for the output voltage across the load capacitance as a function of time was presented and compared to the analysis of the circuit simulator. The results from the governing equation showed very good agreement with the output of the PSPICE simulator as can be seen in Fig. 2.

The solution was also compared to the voltage measurements of the McDonnell Douglas double pulse electronbeam generator (MEDEA II), which was modified from a dual resonance circuit to a triple resonance circuit. Reference 
6 shows several voltage traces during its operation. The circuit parameters were not radically different from the parameters of those of the closed-form solution. Their device showed a gain of 40 . The initial voltage on the primary capacitor was $15.5 \mathrm{kV}$ and the system pulsed a cold fieldemission gun. The pulse-line voltage was compared to the theoretical predictions and the characteristic voltage signature was displayed in the time-dependent pulse-line voltage. There was nothing exhibited in the measured oscillation to raise any questions as to the accuracy and usefulness of the present model.

The structure of Eq. (11) differs from the governing equation of the dual resonance device in basic ways. The dual resonance equation possesses two cosine terms, each representing one of the two coexisting oscillations within the device, ${ }^{1,2}$ with coefficients of unity at all times. Equation (11) contains three cosine terms, each representing one of the coexisting oscillations as before, but the cosine terms now have coefficients that are frequency dependent in a nontrivial manner. These frequency-dependent coefficients will partly define the level of difficulty in optimizing Eq. (11) for maximum voltage gains.

The transmission line between the second and third inductors, labeled TL, deserves attention as it is not properly designed or well understood. The short transmission line must purposely mismatch the two circuits it services. Its design must obtain maximum power transfer, and so demands a conjugate impedance condition. ${ }^{17}$ The complete design of the line will require ingenuity since it also operates at the multimegavolt-level.

The existence of a closed-form equation for the transformer's output voltage now offers the investigators the needed physical insight for future productive work.

One valuable service of the governing equation is to eliminate the incorrect information that begins to surround a device when there is not the slightest guiding physics available to dispel these incorrect notions. Some have designated the magnetically linked inductors as the "transformer section." They maintain, without proof, that the device is capable of any gain by making the ratio of $L_{2} / L_{1}$ as large as required. Equation (11) shows that this is not true, because the ratio is divided by a complicated function of capacitances; that they themselves are functions of the inductances that compose the ratio. In reality, if the ratio is made large, all else held constant, the gain will collapse. Furthermore, a PSPICE run will also confirm this fact.

The decoupled resonate frequency of the third inductor appears to be a controlling factor. It appears to the square power in all the cosine coefficients. A larger primary capacitor was accommodated by manipulating some of the circuit parameters in the demonstrated circuit, and this led to a gain of 50 .

The equation is new, and a deep examination of its functional character has not been carried out.

The present investigator has obtained gains higher than 50 but the modal frequencies were not in whole numbers. There are numerical experiments called for, such as installing the governing equation in an optimizer and studying its output.

In the attempt to obtain higher gains, the governing equation not only reveals how the various parameters compete against each other, but importantly discloses exactly what are the parameters.

\section{ACKNOWLEDGMENT}

Gratitude is extended for the knowledge and work of Terry Fritz of Colorado Springs, Colorado in regard to circuit analysis using OrCAD PSPICE.

${ }^{1}$ P. Drude, Ann. Phys. 13, 512 (1904).

${ }^{2}$ D. Finkelstein, P. Goldberg, and J. Shuchatowitz, Rev. Sci. Instrum. 53, 694 (1966).

${ }^{3}$ E. A. Abramyan, IEEE Trans. Nucl. Sci. NS-18, 447 (1971).

${ }^{4}$ G. J. Rohwein, Sandia Laboratories Report No. SAND79-0813, 1979 (unpublished).

${ }^{5}$ N. Tesla, U.S. Patent No. 1119732 (1 December 1914).

${ }^{6}$ F. M. Bieniosek, Proceedings of the Sixth IEEE Pulsed Power Conference, Arlington, VA, 29 June-1 July 1987 (unpublished).

${ }^{7}$ F. M. Bieniosek, Rev. Sci. Instrum. 61, 1717 (1990).

${ }^{8}$ F. M. Bieniosek, U.S. Patent No. 4833421 (23 May 1989).

${ }^{9}$ A. C. M. de Queiroz, Proceedings of the 2000 IEEE International Symposium on Circuits and Systems (ISCAS), Geneva, Switzerland, 28-31 May 2000 (unpublished).

${ }^{10}$ A. C. M. de Queiroz, www.coe.ufrij.br/ acmq/magnifier.html

${ }^{11}$ M. F. Gardner and J. L. Barnes, Transients in Linear Systems (Wiley, New York, 1961).

${ }^{12}$ A. Erdelyi, Tables of Intergal Transforms (McGraw-Hill, New York, 1954), Vols. I and II.

${ }^{13}$ E. B. Kurtz and G. F. Corcoran, Introduction to Electrical Transients (Wiley, New York, 1953), see Sec. III of the Appendix.

${ }^{14}$ Computer code OrCAD PSPICE (OrCAD, Beverton, OR).

${ }^{15}$ See EPAPS Document No. E-RSINAK-76-238510 for the development of the analytical expression for the output voltage of the triple resonance Tesla transformer and a computer program to exercise the same. This document can be reached through a direct link in the outline article's HTML reference section or via the EPAPS homepage (http://www.aip.org/ pubservs/epaps.html)

${ }^{16}$ Computer code True BASIC; webpage at http://www.truebasic.com

${ }^{17}$ W. Everitt and G. E. Anner, Communication Engineering (McGraw-Hill, New York, 1956), Chap. 11 